\title{
Association of Myopia in Elementary School Students in Jiaojiang District, Taizhou City, China
}

\author{
Xin Lu $(\mathbb{D}),{ }^{1}$ Congcong Guo $\left(\mathbb{D},{ }^{1}\right.$ Bin Xu ${ }^{(D)},{ }^{1}$ Chenwei Hou $\left(\mathbb{D},{ }^{1}\right.$ Xiaoming Huang ${ }^{(D)},{ }^{2}$ Hui Xu $\left(\mathbb{D},{ }^{1}\right.$ \\ and Zhichun Weng $\mathbb{1}^{3}$ \\ ${ }^{1}$ Jiaojiang Center for Disease Control and Prevention, Taizhou, Zhejiang Province, China \\ ${ }^{2}$ Eye Hospital (Taizhou Branch), WMU International Eye Hospital of Taizhou, Taizhou, Zhejiang Province, China \\ ${ }^{3}$ Jiaojiang Education Bureau, Taizhou, Zhejiang Province, China \\ Correspondence should be addressed to Xin Lu; 136198749@qq.com
}

Received 18 July 2020; Revised 13 January 2021; Accepted 20 January 2021; Published 27 February 2021

Academic Editor: Alessandro Meduri

Copyright (c) 2021 Xin Lu et al. This is an open access article distributed under the Creative Commons Attribution License, which permits unrestricted use, distribution, and reproduction in any medium, provided the original work is properly cited.

Background. The aim of our study was to evaluate the prevalence of myopia in elementary school students and to assess the risk factors for myopia. Methods. This school-based cross-sectional study was performed on students from two elementary schools in Jiaojiang, Taizhou City, China. A total of 556 students, whose age ranged from 9 to 12 years, were included. The uncorrected visual acuity and noncycloplegic refractive error tests were performed to determine the myopia. Each student was asked to fulfill the questionnaire about the possible factors associated with myopia. Multivariate logistic analyses of risk factors were conducted. Results. The overall prevalence of myopia among those students was $63.7 \%$, ranged from $53.4 \%$ in grade 4 to $72.5 \%$ in grade 6 . Multivariate logistic analysis showed that adjusting the height of desks and chairs according to the changing height and the presence of myopia in parents were significantly associated with myopia in these students, respectively. Conclusions. Our results showed that myopia among elementary school students was associated with environmental and hereditary factors.

\section{Introduction}

Myopia, also known as short-sightedness, is one of the leading causes of visual disability that develops primarily during childhood when excessive elongation of the eyes results in blurry distance vision and clear close vision [1]. The increasing prevalence of myopia is a global health and social problem [2]. Researchers have estimated that about $50 \%$ of the world's population will be myopic and about $10 \%$ will be high myopic by 2050 [3]. The "myopia boom" is particularly prominent in urban areas of East and Southeast Asia, where $80 \%$ to $90 \%$ of high school graduates have myopia and about $20 \%$ have high myopia $[4,5]$. As the most common visual impairment in children, myopia poses an enormous personal and social burden [6]. Additionally, children with high myopia are at high risk of developing irreversible visual impairment or blindness mostly due to retinal detachment, glaucoma, and myopic macular degeneration $[7,8]$.
Most myopic individuals are associated with excessive axial elongation, and very few occur as a result of disproportionately high corneal power [9]. For adults aged 50 or older, myopia can also be rarely caused by nuclear cataracts [9]. Both environmental and genetic factors impose a significant risk of myopia [10]. The identified genetic variants could explain about $12 \%$ of the variance of the refractive error trait $[11,12]$. Tideman et al. found that different genetic loci were associated with different ages of axial length (AL) and corneal radius (CR) ratio [13]. Among those younger than 10 years, three loci (GJD2, CHRNG, and ZIC2) were associated with AL/CR. In people aged 10 to 25 years, four loci (BMP2, KCNQ5, A2BP1, and CACNA1D) were associated; and in adults (>25 years of age), 20 loci were associated. Environmental factors such as high levels of education, lack of outdoor exposure, and excessive near-work activities are the most established risk factors for myopia [1,5]. A Mendelian 
TABLE 1: Characteristics of students in two elementary schools.

\begin{tabular}{|c|c|c|c|c|c|c|c|c|c|}
\hline \multirow[b]{2}{*}{ Parameters } & \multicolumn{3}{|c|}{ All $(n=556)$} & \multicolumn{3}{|c|}{ School A $(n=310)$} & \multicolumn{3}{|c|}{ School B $(n=246)$} \\
\hline & $\begin{array}{l}\text { Myopia } \\
(n=354)\end{array}$ & $\begin{array}{c}\text { Normal } \\
(n=202)\end{array}$ & $\begin{array}{c}P \\
\text { value }\end{array}$ & $\begin{array}{c}\text { Myopia } \\
(n=201)\end{array}$ & $\begin{array}{c}\text { Normal } \\
(n=109)\end{array}$ & $\begin{array}{c}P \\
\text { value }\end{array}$ & $\begin{array}{c}\text { Myopia } \\
(n=153)\end{array}$ & $\begin{array}{l}\text { Normal } \\
(n=93)\end{array}$ & $\begin{array}{c}P \\
\text { value }\end{array}$ \\
\hline $\begin{array}{l}\text { Age, } \\
\text { mean } \pm S D, y\end{array}$ & $10.21 \pm 0.89$ & $9.89 \pm 0.87$ & $<0.001$ & $10.12 \pm 0.89$ & $9.95 \pm 0.84$ & 0.112 & $10.33 \pm 0.87$ & $9.82 \pm 0.90$ & $<0.001$ \\
\hline \multicolumn{10}{|l|}{ Grade, $n(\%)$} \\
\hline 4 & $102(53.4 \%)$ & $89(46.6 \%)$ & & $68(63.0 \%)$ & $40(37.0 \%)$ & & $34(41.0 \%)$ & $49(59.0 \%)$ & \\
\hline 5 & $120(65.6 \%)$ & $63(34.4 \%)$ & 0.001 & $61(59.8 \%)$ & $41(40.2 \%)$ & 0.169 & $59(72.8 \%)$ & $22(27.2 \%)$ & $<0.001$ \\
\hline 6 & $132(72.5 \%)$ & $50(27.5 \%)$ & & $72(72.0 \%)$ & $28(28.0 \%)$ & & $60(73.2 \%)$ & $22(26.8 \%)$ & \\
\hline \multicolumn{10}{|l|}{ Gender, $n(\%)$} \\
\hline Male & $194(63.2 \%)$ & $113(36.8 \%)$ & & $110(63.6 \%)$ & $63(36.4 \%)$ & & $84(62.7 \%)$ & $50(37.3 \%)$ & 0.862 \\
\hline Female & $160(64.3 \%)$ & $89(35.7 \%)$ & 0.795 & $91(66.4 \%)$ & $46(33.6 \%)$ & 0.603 & $69(61.6 \%)$ & $43(38.4 \%)$ & 0.862 \\
\hline
\end{tabular}

randomization study by Mountjoy et al. also showed that more time in education may be a causal risk factor for myopia [14]. Since refractive error correction could not prevent the myopic pathologies, preventing the myopia and particularly high myopia at the early age is of great significance $[1,4]$. Each year of delay in the age at onset could substantially reduce the chance of developing high myopia in adulthood [15]. With the aim of discovering potentially effective prevention methods during childhood, in this cross-sectional study, we collected children in elementary schools to evaluate the prevalence of myopia in these young populations and assess the protective and risk factors for myopia.

\section{Materials and Methods}

Two elementary schools (school A and school B) in Jiaojiang District, Taizhou City, Zhejiang Province, China, were included. Students from grades 4 to 6 were enrolled from September to October 2019. Two or three classes were randomly selected in each grade, and all students in selected classes were enrolled.

Each participant was asked to fulfill the customized questionnaire, including the characteristics of students and possible factors associated with myopia. The uncorrected visual acuity (UCVA) and noncycloplegic refractive error tests were performed by pediatric ophthalmologists from Taizhou Municipal Hospital. The UCVA was tested using the Standard Logarithmic Visual Acuity E Chart, and noncycloplegic refractive error was tested using the RM-800 Auto Refractometer (Topcon Medical Systems, Inc). The UCVA less than 5.0 and spherical equivalent refraction less than -0.50 diopter in at least one eye were used to define the myopia.

Statistical analyses were conducted using the Statistical Package for the Social Sciences (SPSS, version 21.0; IBM, Chicago). The chi-squared tests were used to evaluate the associations between factors and myopia. The parameters with a univariate association were selected as candidate variates for multivariate logistic analysis. The odds ratio (OR) and 95\% confidence intervals (CIs) were calculated. A $P$ value of less than 0.05 was considered statistically significant.

\section{Results}

A total of 556 students ( 310 in school A and 246 in school B) were included in this study. The prevalence of myopia was $63.7 \%$, with $64.8 \%$ in school A and $62.2 \%$ in school B. There is no statistical difference in the prevalence of myopia between the two schools $(P=0.520)$. The prevalence of myopia showed statistically different among grade 4 , grade 5 , and grade 6 in school $\mathrm{B}(P<0.001)$ and total $(P=0.001)$, respectively. The average age of students with myopia was higher than those of normal students in school B and total (both $P<0.001)$. No statistical difference in the proportion of myopia was found between males and females (Table 1).

Table 2 shows the associations between factors studied and the prevalence of myopia in primary school students. The frequencies of changing class seats and adjusting the height of desks and chairs were statistically associated with the presence of myopia $(P<0.05)$. Sleeping time more than $8 \mathrm{~h}$ and the presence of myopia in parents were also found to be associated with the prevalence of myopia $(P<0.05)$. No other factor showed a univariate association.

After adjusting the age and gender, adjusting the height of desks and chairs according to the changing height and the presence of myopia in parents were still associated with the presence of myopia (all $P<0.05$, Table 3 ). Comparing with never adjusting the height of desks and chairs, adjusting the height of desks and chairs once a year and once a semester in total $(\mathrm{OR}=0.37,95 \% \mathrm{CI}=0.21-0.67, P=0.001$; $\mathrm{OR}=0.60$, $95 \% \mathrm{CI}=0.35-0.97, P=0.037)$ and adjusting the height of desks and chairs once a year in school $\mathrm{B}(\mathrm{OR}=0.26,95 \%$ $\mathrm{CI}=0.11-0.62, P=0.003)$ were protective factors. Parents having no myopia was a protective factor for myopia in total $(\mathrm{OR}=0.51, \quad 95 \% \quad \mathrm{CI}=0.35-0.74, \quad P<0.001)$, school $\mathrm{A}$ $(\mathrm{OR}=0.56,95 \% \mathrm{CI}=0.34-0.93, P=0.026)$, and school $\mathrm{B}$ (OR $=0.45,95 \% \mathrm{CI}=0.25-0.83, P=0.009)$, respectively.

\section{Discussion}

In this study, we identified that adjusting the height of desks and chairs according to the changing height and the presence of myopia in parents were associated with myopia in elementary school students.

The prevalence of myopia in our study was $63.7 \%$, which was similar to the myopia prevalence of $66.5 \%$ among 
TABLE 2: The associations between factors and the prevalence of myopia.

\begin{tabular}{|c|c|c|c|c|c|c|c|c|c|}
\hline \multirow[b]{2}{*}{ Parameters } & \multicolumn{3}{|c|}{ All $(n=556)$} & \multicolumn{3}{|c|}{ School A $(n=310)$} & \multicolumn{3}{|c|}{ School B $(n=246)$} \\
\hline & $\begin{array}{l}\text { Myopia } \\
(n=354)\end{array}$ & $\begin{array}{c}\text { Normal } \\
(n=202)\end{array}$ & $\begin{array}{c}P \\
\text { value }\end{array}$ & $\begin{array}{l}\text { Myopia } \\
(n=201) \\
\end{array}$ & $\begin{array}{c}\text { Normal } \\
(n=109)\end{array}$ & $\begin{array}{c}P \\
\text { value }\end{array}$ & $\begin{array}{l}\text { Myopia } \\
(n=153)\end{array}$ & $\begin{array}{l}\text { Normal } \\
(n=93)\end{array}$ & $\begin{array}{c}P \\
\text { value }\end{array}$ \\
\hline \multicolumn{10}{|c|}{ Change class seats, $n(\%)$} \\
\hline Never & $0(0.0 \%)$ & $5(100.0 \%)$ & \multirow{5}{*}{0.030} & $0(0.0 \%)$ & $3(100.0 \%)$ & & $0(0.0 \%)$ & $2(100.0 \%)$ & \multirow{5}{*}{0.202} \\
\hline Once a semester & $5(55.6 \%)$ & $4(44.4 \%)$ & & $2(50.0 \%)$ & $2(50.0 \%)$ & & $3(60.0 \%)$ & $2(40.0 \%)$ & \\
\hline Once a month & $49(65.3 \%)$ & $26(34.7 \%)$ & & $43(68.3 \%)$ & $20(31.7 \%)$ & 0.115 & $6(50.0 \%)$ & $6(50.0 \%)$ & \\
\hline Once a fortnight & $256(64.0 \%)$ & $144(36.0 \%)$ & & $145(65.6 \%)$ & $76(34.4 \%)$ & & $111(62.0 \%)$ & $68(38.0 \%)$ & \\
\hline Once a week & $44(66.7 \%)$ & $22(33.3 \%)$ & & $11(57.9 \%)$ & $8(42.1 \%)$ & & $33(70.2 \%)$ & $14(29.8 \%)$ & \\
\hline \multicolumn{10}{|c|}{ Adjust the height of desks and chairs, $n(\%)$} \\
\hline Never & $88(66.7 \%)$ & $44(33.3 \%)$ & \multirow{4}{*}{0.008} & $37(61.7 \%)$ & $23(38.3 \%)$ & \multirow{4}{*}{0.629} & $51(70.8 \%)$ & $21(29.2 \%)$ & \multirow{4}{*}{$<0.001$} \\
\hline Once a year & $44(48.4 \%)$ & $47(51$. & & $19(70.4 \%)$ & $8(29.8 \%)$ & & $25(39.1 \%)$ & $39(60.9 \%)$ & \\
\hline Once a semester & $176(65.9 \%)$ & $91(34.1$ & & $104(63.0 \%)$ & $61(37.0 \%)$ & & $72(70.6 \%)$ & $30(29.4 \%)$ & \\
\hline $\begin{array}{l}\text { Once every } 2 \text { to } 3 \\
\text { months }\end{array}$ & $46(70.8 \%)$ & $19(29.2 \%)$ & & $41(70.7 \%)$ & $17(29.3 \%)$ & & $5(71.4 \%)$ & $2(28.6 \%)$ & \\
\hline \multicolumn{10}{|c|}{ Activity place during recess, $n(\%)$} \\
\hline Teaching building & $264(63.9 \%)$ & $149(36.1 \%)$ & \multirow[b]{2}{*}{0.908} & $153(66.5 \%)$ & $77(33.5 \%)$ & \multirow[b]{2}{*}{0.293} & $111(60.7 \%)$ & $72(39.3 \%)$ & \multirow[b]{2}{*}{0.319} \\
\hline $\begin{array}{l}\text { Outside teaching } \\
\text { building }\end{array}$ & $90(63.4 \%)$ & $52(36.6 \%)$ & & $48(60.0 \%)$ & $32(40.0 \%)$ & & $42(67.7 \%)$ & $20(32.3 \%)$ & \\
\hline \multicolumn{10}{|c|}{ Time for homework per day, $n(\%)$} \\
\hline$<1 \mathrm{~h}$ & $52(65.0 \%)$ & $28(35.0 \%)$ & & $48(69.6 \%)$ & $21(30.4 \%)$ & & $4(36.4 \%)$ & $7(63.6 \%)$ & \\
\hline $1-1.99 \mathrm{~h}$ & $\%)$ & & & & 45 & & & 47 & \\
\hline $2-2.99 \mathrm{~h}$ & $106(65$. & $56(34.6 \%)$ & 0.828 & $47(63.5 \%)$ & $27(36.5 \%)$ & 0.196 & $59(67.0$ & $29(33.0 \%)$ & 0.280 \\
\hline$\geq 3 \mathrm{~h}$ & $37(62.7 \%)$ & $22(37.2 \%)$ & & $18(56.3 \%)$ & $14(43.8 \%)$ & & $19(70.4 \%)$ & $8(29.6 \%)$ & \\
\hline Uncertain & $2(40.0 \%)$ & $3(60.0 \%)$ & & $0(0.0 \%)$ & $2(100.0 \%)$ & & $2(66.7 \%)$ & & \\
\hline Time for interest clo & her unok n & & & & & & & & \\
\hline $0 \mathrm{~h}$ & $\%)$ & $38(40.0 \%)$ & & $33(67.3 \%)$ & $16(32.7 \%)$ & & & $22(47.8 \%)$ & \\
\hline$<1 \mathrm{~h}$ & & & & & & & & & \\
\hline $1-1.99 \mathrm{~h}$ & $66(64.7 \%)$ & $36(35.3 \%)$ & & $43(67.2 \%)$ & $21(32.8 \%)$ & & $23(60.5 \%)$ & $15(39.5 \%)$ & \\
\hline $2-2.99 \mathrm{~h}$ & $81(62.3 \%)$ & $49(37.7 \%)$ & 0.862 & $37(59.7 \%)$ & $25(40.3 \%)$ & 0.789 & $44(64.7 \%)$ & $24(35.3 \%)$ & 0.665 \\
\hline$\geq 3 \mathrm{~h}$ & $129(66.2 \%)$ & $66(33.8 \%)$ & & $73(66.4 \%)$ & $37(33.6 \%)$ & & & $29(34.1 \%)$ & \\
\hline Uncertain & $4(80.0 \%)$ & & & & & & & & \\
\hline Parents limit sports & for study, $n$ & & & & & & & & \\
\hline Often & 28 & 18 & & $17(65.4 \%)$ & $9(34.6 \%)$ & & $11(55.0 \%)$ & $9(45.0 \%)$ & \\
\hline Sometimes & $111(65.7 \%)$ & $58(34.3 \%)$ & 0.788 & $60(64.5 \%)$ & $33(3$ & 0.996 & $51(67.1 \%)$ & $25(32.9 \%)$ & 0.523 \\
\hline Never & $215(63.2 \%)$ & $125(36.8 \%)$ & & $124(64.9 \%)$ & $67(35.1 \%)$ & & & $58(38.9 \%)$ & \\
\hline Parents limit electro & $1 \quad 10$ & & & & & & & & \\
\hline Yes & $3 \%)$ & $179(35.7 \%)$ & & $182(66.2 \%)$ & $93(33.8 \%)$ & & & & \\
\hline No & $31(58.5 \%)$ & $22(41.5 \%)$ & c & $19(54.3 \%)$ & $16(45.7 \%)$ & & $12(66.7 \%)$ & $6(33.3 \%)$ & 0.7 \\
\hline Sit more than one-pu & distoro fro & the do of t & Chond & ho reding & & & & & \\
\hline Never & $23(59.0 \%)$ & $16(41.0 \%)$ & & $15(57.7 \%)$ & $11(42.3 \%)$ & & $8(61.5 \%)$ & $5(38.5 \%)$ & \\
\hline Sometimes & $108(62.1 \%)$ & $66(37.9 \%)$ & & $57(62.6 \%)$ & $34(37.4 \%)$ & & $51(61.4 \%)$ & $32(38.6 \%)$ & \\
\hline Often & $129(62.9 \%)$ & $76(37.1 \%)$ & .560 & $56(63.6 \%)$ & $32(36.4 \%)$ & 0.606 & $73(62.4 \%)$ & $44(37.6 \%)$ & 0.981 \\
\hline Always & $94(68.6 \%)$ & $43(31.4 \%)$ & & $73(69.5 \%)$ & $32(30.5 \%)$ & & $21(65.6 \%)$ & $11(34.4 \%)$ & \\
\hline The distance betwee & s and hooks & morethan 3 & 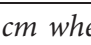 & moding an & vriting, $n(\%)$ & & & & \\
\hline Never & $21(53.8 \%)$ & $18(46.2 \%)$ & & $15(57.7 \%)$ & $11(42.3 \%)$ & & $7(50.0 \%)$ & $7(50.0 \%)$ & \\
\hline Sometimes & $114(62.3 \%)$ & $69(37.7 \%)$ & & $57(62.6 \%)$ & $34(37.4 \%)$ & & $52(66.7 \%)$ & $26(33.3 \%)$ & \\
\hline Often & $137(63.4 \%)$ & $79(36.6 \%)$ & .270 & $56(63.6 \%)$ & $32(36.4 \%)$ & 0.606 & $79(61.2 \%)$ & $50(38.8 \%)$ & 0.660 \\
\hline Always & $82(70.1 \%)$ & $35(29.9 \%)$ & & $73(69.5 \%)$ & $32(30.5 \%)$ & & & & \\
\hline The distance betwee & are and nib & & & $1 . \quad 1 \quad 1$ & & & & & \\
\hline Never & $31(53.4 \%)$ & $27(46.6 \%)$ & & $15(53.6 \%)$ & $13(46.4 \%)$ & & $16(53.3 \%)$ & $14(46.7 \%)$ & \\
\hline Sometimes & $65(61.9 \%)$ & $40(38.1 \%)$ & & $38(61.3 \%)$ & $24(38.7 \%)$ & & $27(62.8 \%)$ & $16(37.2 \%)$ & \\
\hline Often & $125(61.3 \%)$ & $79(38.7 \%)$ & & $44(57.9 \%)$ & $32(42.1 \%)$ & 0.073 & $81(63.3 \%)$ & $47(36.7 \%)$ & \\
\hline Always & $133(70.7 \%)$ & $55(29.3 \%)$ & & $104(72.2 \%)$ & $40(27.8 \%)$ & & $29(65.9 \%)$ & $15(34.1 \%)$ & \\
\hline Teachers remind th & $f$ li & & & & & & & & \\
\hline Never & $28(65.1 \%)$ & $15(34.9 \%)$ & & & $9(33.3 \%)$ & & & $6(37.5 \%)$ & \\
\hline Sometimes & $75(56.4 \%)$ & $58(43.6 \%)$ & & $46(61.3 \%)$ & $29(38.7 \%)$ & & $29(50.0 \%)$ & $29(50.0 \%)$ & \\
\hline Often & $90(64.7 \%)$ & $49(35.3 \%)$ & ( & $47(65.3 \%)$ & $25(34.7 \%)$ & 0.906 & $43(64.2 \%)$ & $24(35.8 \%)$ & \\
\hline Always & $161(67.1 \%)$ & $79(32.9 \%)$ & & $90(66.2 \%)$ & $46(33.8 \%)$ & & $71(68.3 \%)$ & $33(31.7 \%)$ & \\
\hline
\end{tabular}


TABle 2: Continued.

\begin{tabular}{|c|c|c|c|c|c|c|c|c|c|}
\hline \multirow[b]{2}{*}{ Parameters } & \multicolumn{3}{|c|}{ All $(n=556)$} & \multicolumn{3}{|c|}{ School A $(n=310)$} & \multicolumn{3}{|c|}{ School B $(n=246)$} \\
\hline & $\begin{array}{l}\text { Myopia } \\
(n=354)\end{array}$ & $\begin{array}{l}\text { Normal } \\
(n=202)\end{array}$ & $\begin{array}{c}P \\
\text { value }\end{array}$ & $\begin{array}{l}\text { Myopia } \\
(n=201)\end{array}$ & $\begin{array}{l}\text { Normal } \\
(n=109)\end{array}$ & $\begin{array}{c}P \\
\text { value }\end{array}$ & $\begin{array}{l}\text { Myopia } \\
(n=153)\end{array}$ & $\begin{array}{l}\text { Normal } \\
(n=93)\end{array}$ & $\begin{array}{c}P \\
\text { value }\end{array}$ \\
\hline \multicolumn{10}{|c|}{ Parents remind the gestures of reading and writing, $n(\%)$} \\
\hline Never & $13(48.1 \%)$ & $14(51.9 \%)$ & \multirow{4}{*}{0.145} & $9(50.0 \%)$ & $9(50.0 \%)$ & \multirow{4}{*}{0.379} & $4(44.4 \%)$ & $5(55.6 \%)$ & \multirow{4}{*}{0.462} \\
\hline Sometimes & $60(64.5 \%)$ & $33(35.5 \%)$ & & $37(67.3 \%)$ & $18(32.7 \%)$ & & $23(60.5 \%)$ & $15(39.5 \%)$ & \\
\hline Often & $108(60.3 \%)$ & $71(39.7 \%)$ & & $55(61.1 \%)$ & $35(38.9 \%)$ & & $53(59.6 \%)$ & $36(40.4 \%)$ & \\
\hline Always & $173(67.6 \%)$ & $83(32.4 \%)$ & & $100(68.0 \%)$ & $47(32.0 \%)$ & & $73(67.0 \%)$ & $36(33.0 \%)$ & \\
\hline \multicolumn{10}{|c|}{ Watching TV per day, $n(\%)$} \\
\hline Never & $51(66.2 \%)$ & $26(33.8 \%)$ & \multirow{6}{*}{0.085} & $33(68.8 \%)$ & $15(31.3 \%)$ & \multirow{6}{*}{0.051} & $18(62.1 \%)$ & $11(37.9 \%)$ & \multirow{6}{*}{0.494} \\
\hline$<1 \mathrm{~h}$ & $187(61.5 \%)$ & $117(38.5 \%)$ & & $93(61.6 \%)$ & $58(38.4 \%)$ & & $94(61.4 \%)$ & $59(38.6 \%)$ & \\
\hline $1-1.99 \mathrm{~h}$ & $86(69.4 \%)$ & $38(30.6 \%)$ & & $56(69.1 \%)$ & $25(30.9 \%)$ & & $30(69.8 \%)$ & $13(30.2 \%)$ & \\
\hline $2-2.99 \mathrm{~h}$ & $21(72.4 \%)$ & $8(27.6 \%)$ & & $14(87.5 \%)$ & $2(12.5 \%)$ & & $7(53.8 \%)$ & $6(46.2 \%)$ & \\
\hline $3-3.99 \mathrm{~h}$ & $3(75.0 \%)$ & $1(25.0 \%)$ & & $1(50.0 \%)$ & $1(50.0 \%)$ & & $2(100.0 \%)$ & $0(0.0 \%)$ & \\
\hline$\geq 4 \mathrm{~h}$ & $6(35.3 \%)$ & $11(64.7 \%)$ & & $4(33.3 \%)$ & $8(66$. & & $2(40.0 \%)$ & $3(60$ & \\
\hline \multicolumn{10}{|c|}{ Using computers per day, $n(\%)$} \\
\hline Never $T$ & $190(64.0 \%)$ & $107(36.0 \%)$ & \multirow{6}{*}{0.250} & $122(66.7 \%)$ & $61(33.3 \%)$ & \multirow{6}{*}{0.221} & $68(59.6 \%)$ & $46(40.4 \%)$ & \multirow{6}{*}{0.637} \\
\hline$<1 \mathrm{~h}$ & $138(65.4 \%)$ & $73(34.6 \%)$ & & $57(65.5 \%)$ & $30(34$ & & $81(65.3 \%)$ & $43(34$ & \\
\hline $1-1.99 \mathrm{~h}$ & $21(52.5 \%)$ & $19(47.5 \%)$ & & $17(51.5 \%)$ & $16(48.5 \%)$ & & $4(57.1 \%)$ & $3(42.9 \%)$ & \\
\hline $2-2.99 \mathrm{~h}$ & $3(100.0 \%)$ & $0(0.0 \%)$ & & $3(100.0 \%)$ & $0(0.0 \%)$ & & 0 & 0 & \\
\hline $3-3.99 \mathrm{~h}$ & $2(50.0 \%)$ & $2(50.0 \%)$ & & 0 & 0 & & 0 & 0 & \\
\hline$\geq 4 \mathrm{~h}$ & & & & $2(50.0 \%)$ & $2(50.0 \%)$ & & 0 & 0 & \\
\hline \multicolumn{10}{|c|}{ Using mobile devices more than 1 hour per day, $n(\%)$} \\
\hline Yes & $278(63.3 \%)$ & $161(36.7 \%)$ & & $48(63.2 \%)$ & $28(36.8 \%)$ & & $28(68.3 \%)$ & $13(31.7 \%)$ & \\
\hline No & $76(65.0 \%)$ & $41(35.0 \%)$ & 0.744 & $153(65.4 \%)$ & $81(34.6 \%)$ & 0.724 & $125(61.0 \%)$ & $80(39.0 \%)$ & $0.3 / 8$ \\
\hline Reading books or electr & nic screens in & lirect sunlight, & $n(\%)$ & & & & & & \\
\hline Never & $264(62.3 \%)$ & $160(37.7 \%)$ & & $159(64.9 \%)$ & $86(35.1 \%)$ & & $105(58.7 \%)$ & $74(41.3 \%)$ & \\
\hline Sometimes & $79(68.7 \%)$ & $36(31.3 \%)$ & & $33(63.5 \%)$ & $19(36.5 \%)$ & & $46(73.0 \%)$ & $17(27.0 \%)$ & \\
\hline Often & $4(50.0 \%)$ & $4(50.0 \%)$ & & $3(50.0 \%)$ & $3(50.0 \%)$ & 0.5 & $1(50.0 \%)$ & $1(50.0 \%)$ & \\
\hline Always & $7(87.5 \%)$ & $1(12.5 \%)$ & & $6(85.7 \%)$ & $1(14.3 \%)$ & & $1(100.0 \%)$ & $0(0.0 \%)$ & \\
\hline Turn off the light whe & looking at the & lectronic screo & after & lark, $n(\%)$ & & & & & \\
\hline Never & $282(63.5 \%)$ & $162(36.5 \%)$ & & $171(65.0 \%)$ & $92(35.0 \%)$ & & & & \\
\hline Sometimes & $61(66$ & $31(3$ & & $21(63.6 \%)$ & $12(36.4 \%)$ & & $40(67.8 \%)$ & $19(32.2 \%)$ & \\
\hline Often & $5(55.6 \%)$ & $4(44.4 \%)$ & 0.899 & $4(66.7 \%)$ & $2(33.3 \%)$ & 0.997 & $1(33.3 \%)$ & $2(66.7 \%)$ & 0.572 \\
\hline Always & $6(60.0 \%)$ & $4(40.0 \%)$ & & $5(62.5 \%)$ & $3(37.5 \%)$ & & $1(50.0 \%)$ & $1(50.0 \%)$ & \\
\hline Reading or looking at & ctronic screer & by lying, $n$ & & & & & & & \\
\hline Never & $189(64.3 \%)$ & $105(35.7 \%)$ & & $121(65.4 \%)$ & $64(34.6 \%)$ & & $68(62.4 \%)$ & $41(37.6 \%)$ & \\
\hline Sometimes & $9(62.9 \%)$ & $76(37.1 \%)$ & & $64(64.0 \%)$ & $36(36.0 \%)$ & & $65(61.9 \%)$ & $40(38.1 \%)$ & \\
\hline Often & $31(62.0 \%)$ & $19(38.0 \%)$ & 0.732 & $12(57.1 \%)$ & $9(42.9 \%)$ & 0.256 & $19(65.5 \%)$ & $10(34.5 \%)$ & 0.968 \\
\hline Always & $5(83.3 \%)$ & $1(16.7 \%)$ & & $4(100.0 \%)$ & $0(0.0 \%)$ & & $1(50.0 \%)$ & $1(50.0 \%)$ & \\
\hline Reading or looking at & ectronic screen & when walkin & or tak & $n g$ a bus, $n(\%$ & & & & & \\
\hline Never & $278(63.2 \%)$ & $162(36.8 \%)$ & & $165(65.5 \%)$ & $87(34.5 \%)$ & & $113(60.1 \%)$ & $75(39.9 \%)$ & \\
\hline Sometimes & $71(65.7 \%)$ & $37(34.3 \%)$ & & $33(61.1 \%)$ & $21(38.9 \%)$ & & $38(70.4 \%)$ & $16(29.6 \%)$ & \\
\hline Often & $5(71.4 \%)$ & $2(28.6 \%)$ & 0.805 & $3(75.0 \%)$ & $1(25.0 \%)$ & 0.756 & $2(66.7 \%)$ & $1(33.3 \%)$ & 0.376 \\
\hline Always & 0 & 0 & & 0 & 0 & & 0 & 0 & \\
\hline The lamp used when re & ading after dar & $(\%)$ & & & & & & & \\
\hline $\begin{array}{l}\text { Both desk lamp and } \\
\text { roof lamp }\end{array}$ & $230(65.2 \%)$ & $123(34.8 \%)$ & & $125(64.8 \%)$ & $68(35.2 \%)$ & & $105(65.6 \%)$ & $55(34.4 \%)$ & \\
\hline Only desk lamp & $24(53.3 \%)$ & $21(46.7 \%)$ & 0.354 & $14(50.0 \%)$ & $14(50.0 \%)$ & 0.237 & & & 0.362 \\
\hline Only roof lamp & $99(63.5 \%)$ & $57(36.5 \%)$ & & $61(69.3 \%)$ & $27(30.7 \%)$ & & $38(55.9 \%)$ & $30(44.1 \%)$ & \\
\hline Others & $1(100.0 \%)$ & $0(0.0 \%)$ & & $1(100.0 \%)$ & $0(0.0 \%)$ & & 0 & 0 & \\
\hline The distance between e & s and screens & more than 66 & n whe & using compu & $n(\%)$ & & & & \\
\hline $\begin{array}{l}\text { Never using } \\
\text { computers }\end{array}$ & $115(68.9 \%)$ & $52(31.1 \%)$ & & $77(73.3 \%)$ & $28(26.7 \%)$ & & $38(61.3 \%)$ & $24(38.7 \%)$ & \\
\hline Never & & & & & $10(45.5 \%)$ & & $22(62.9 \%)$ & $13(37.1 \%)$ & \\
\hline Sometimes & $72(62.6 \%)$ & $43(37.4 \%)$ & 0.483 & $26(63.4 \%)$ & $15(36.6 \%)$ & 0.238 & $46(62.2 \%)$ & $28(37.8 \%)$ & 0.844 \\
\hline Often & $57(64.8 \%)$ & $31(35.2 \%)$ & & $23(60.5 \%)$ & $15(39.5 \%)$ & & $34(68.0 \%)$ & $16(32.0 \%)$ & \\
\hline Always & $76(59.4 \%)$ & $52(40.6 \%)$ & & $63(60.6 \%)$ & $41(39.4 \%)$ & & $13(54.2 \%)$ & $11(45.8 \%)$ & \\
\hline
\end{tabular}


TABle 2: Continued.

\begin{tabular}{|c|c|c|c|c|c|c|c|c|c|}
\hline \multirow[b]{2}{*}{ Parameters } & \multicolumn{3}{|c|}{ All $(n=556)$} & \multicolumn{3}{|c|}{ School A $(n=310)$} & \multicolumn{3}{|c|}{ School B $(n=246)$} \\
\hline & $\begin{array}{c}\text { Myopia } \\
(n=354)\end{array}$ & $\begin{array}{c}\text { Normal } \\
(n=202)\end{array}$ & $\begin{array}{c}P \\
\text { value }\end{array}$ & $\begin{array}{c}\text { Myopia } \\
(n=201)\end{array}$ & $\begin{array}{c}\text { Normal } \\
(n=109)\end{array}$ & $\begin{array}{c}P \\
\text { value }\end{array}$ & $\begin{array}{c}\text { Myopia } \\
(n=153)\end{array}$ & $\begin{array}{l}\text { Normal } \\
(n=93)\end{array}$ & $\begin{array}{c}P \\
\text { value }\end{array}$ \\
\hline \multicolumn{10}{|c|}{ The distance between eyes and TV more than $3 \mathrm{~m}$ when watching $T V, n(\%)$} \\
\hline Never watching TV & $28(65.1 \%)$ & $15(34.9 \%)$ & \multirow{5}{*}{0.319} & $17(70.8 \%)$ & $7(29.2 \%)$ & \multirow{5}{*}{0.318} & $11(57.9 \%)$ & $8(42.1 \%)$ & \multirow{5}{*}{0.123} \\
\hline Never & $27(62.8 \%)$ & $16(37.2 \%)$ & & $13(52.0 \%)$ & $12(48.0 \%)$ & & $14(77.8 \%)$ & $4(22.2 \%)$ & \\
\hline Sometimes & $101(70.6 \%)$ & $42(29.4 \%)$ & & $39(70.9 \%)$ & $16(29.1 \%)$ & & $62(70.5 \%)$ & $26(29.5 \%)$ & \\
\hline Often & $72(63.2 \%)$ & $42(36.8 \%)$ & & $45(70.3 \%)$ & $19(29.7 \%)$ & & $27(54.0 \%)$ & $23(46.0 \%)$ & \\
\hline Always & $126(59.4 \%)$ & $86(40.6 \%)$ & & $87(61.3 \%)$ & $55(38.7 \%)$ & & $39(55.7 \%)$ & $31(44.3 \%)$ & \\
\hline \multicolumn{10}{|c|}{ Time on outdoor activities at daytime per day, $n(\%)$} \\
\hline$<1 \mathrm{~h}$ & $60(59.4 \%)$ & $41(40.6 \%)$ & \multirow{5}{*}{0.130} & $39(62.9 \%)$ & $23(37.1 \%)$ & \multirow{5}{*}{0.276} & $21(53.8 \%)$ & $18(46.2 \%)$ & \multirow{5}{*}{0.343} \\
\hline $1-1.99 \mathrm{~h}$ & $184(68.9 \%)$ & $83(31.1 \%)$ & & $110(69.2 \%)$ & $49(30.8 \%)$ & & $74(68.5 \%)$ & $34(31.5 \%)$ & \\
\hline $2-2.99 \mathrm{~h}$ & $61(62.2 \%)$ & $37(37.8 \%)$ & & $23(67.6 \%)$ & $11(32.4 \%)$ & & $38(59.4 \%)$ & $26(40.6 \%)$ & \\
\hline$\geq 3 \mathrm{~h}$ & $40(54.1 \%)$ & $34(45.9 \%)$ & & $24(53.3 \%)$ & $21(46.7 \%)$ & & $16(55.2 \%)$ & $13(44.8 \%)$ & \\
\hline Uncertain & $9(60.0 \%)$ & $6(40.0 \%)$ & & $5(50.0 \%)$ & $5(50.0 \%)$ & & $4(80.0 \%)$ & $1(20.0 \%)$ & \\
\hline \multicolumn{10}{|c|}{ Sleeping time more than $8 h, n(\%)$} \\
\hline Yes & $346(64.6 \%)$ & $190(35.4 \%)$ & \multirow{2}{*}{0.025} & $197(66.1 \%)$ & $101(33.9 \%)$ & \multirow{2}{*}{0.043} & $149(62.6 \%)$ & $89(37.4 \%)$ & \multirow{2}{*}{0.72} \\
\hline No & $8(40.0 \%)$ & $12(60.0 \%)$ & & $4(33.3 \%)$ & $8(66.7 \%)$ & & $4(50.0 \%)$ & $4(50.0 \%)$ & \\
\hline \multicolumn{10}{|c|}{ Father or mother has myopia, $n(\%)$} \\
\hline Yes & $222(69.4 \%)$ & $98(30.6 \%)$ & \multirow{2}{*}{0.001} & $120(70.6 \%)$ & $50(29.4 \%)$ & \multirow{2}{*}{0.019} & $102(68.0 \%)$ & $48(32.0 \%)$ & \multirow{2}{*}{0.024} \\
\hline No & $132(56.2 \%)$ & $103(43.8 \%)$ & & $81(57.9 \%)$ & $59(42.1 \%)$ & & $51(53.7 \%)$ & $44(46.3 \%)$ & \\
\hline \multicolumn{10}{|c|}{ Performed the examination of myopia in the past year, $n(\%)$} \\
\hline Yes & $336(64.2 \%)$ & $187(35.8 \%)$ & \multirow{2}{*}{0.361} & & & \multirow{2}{*}{0.444} & & & \multirow{2}{*}{0.814} \\
\hline No & $18(56.3 \%)$ & $14(43.8 \%)$ & & $12(57.1 \%)$ & $9(42.9 \%)$ & & $6(54.5 \%)$ & $5(45.5 \%)$ & \\
\hline
\end{tabular}

TABLE 3: The associations between factors and myopia using multivariate logistic regression.

\begin{tabular}{|c|c|c|c|c|c|c|}
\hline \multirow{2}{*}{ Parameters } & \multicolumn{2}{|c|}{ All $(n=556)$} & \multicolumn{2}{|c|}{ School A $(n=310)$} & \multicolumn{2}{|c|}{ School B $(n=246)$} \\
\hline & OR $(95 \% \mathrm{CI})$ & $P$ value & OR (95\% CI) & $P$ value & OR $(95 \% \mathrm{CI})$ & $P$ value \\
\hline Age & $1.57(0.87,2.80)$ & 0.131 & $1.47(0.55,3.92)$ & 0.437 & $1.75(0.82,3.75)$ & 0.149 \\
\hline \multicolumn{7}{|l|}{ Grade } \\
\hline 4 & $0.94(0.26,3.37)$ & 0.919 & $1.20(0.15,9.91)$ & 0.866 & $0.97(0.16,6.08)$ & 0.976 \\
\hline 5 & $1.03(0.48,2.21)$ & 0.936 & $0.88(0.27,2.91)$ & 0.837 & $1.28(0.39,4.16)$ & 0.686 \\
\hline 6 & Reference & & Reference & & Reference & \\
\hline \multicolumn{7}{|l|}{ Gender } \\
\hline Male & $0.98(0.68,1.42)$ & 0.914 & $0.87(0.53,1.44)$ & 0.596 & $1.08(0.60,1.95)$ & 0.795 \\
\hline Female & Reference & & Reference & & Reference & \\
\hline \multicolumn{7}{|l|}{ Change class seats } \\
\hline Never & - & - & - & - & - & \\
\hline Once a semester & $1.09(0.25,4.82)$ & 0.907 & $0.83(0.09,7.82)$ & 0.867 & $1.07(0.13,8.73)$ & 0.950 \\
\hline Once a month & $1.08(0.52,2.26)$ & 0.829 & $1.17(0.38,3.64)$ & 0.781 & $0.58(0.12,2.75)$ & 0.497 \\
\hline Once a fortnight & $1.10(0.61,2.01)$ & 0.748 & $1.23(0.43,3.52)$ & 0.694 & $1.15(0.50,2.64)$ & 0.749 \\
\hline Once a week & Reference & & Reference & & Reference & \\
\hline \multicolumn{7}{|c|}{ Adjust the height of desks and chairs } \\
\hline Never & Reference & & Reference & & Reference & \\
\hline Once a year & $0.37(0.21,0.67)$ & 0.001 & $1.07(0.36,3.13)$ & 0.908 & $0.26(0.11,0.62)$ & 0.003 \\
\hline Once a semester & $0.60(0.35,0.97)$ & 0.037 & $0.74(0.34,1.60)$ & 0.450 & $0.55(0.22,1.34)$ & 0.188 \\
\hline Once every 2 to 3 months & $0.76(0.37,1.56)$ & 0.452 & $0.98(0.38,2.52)$ & 0.973 & $0.82(0.13,5.13)$ & 0.827 \\
\hline \multicolumn{7}{|l|}{ Sleeping time more than $8 \mathrm{~h}$} \\
\hline Yes & Reference & & Reference & & Reference & \\
\hline No & $0.45(0.17,1.18)$ & 0.103 & $0.29(0.08,1.03)$ & 0.055 & $1.15(0.21,6.18)$ & 0.870 \\
\hline \multicolumn{7}{|l|}{ Father or mother has myopia } \\
\hline Yes & Reference & & Reference & & Reference & \\
\hline No & $0.51(0.35,0.74)$ & $<0.001$ & $0.56(0.34,0.93)$ & 0.026 & $0.45(0.25,0.83)$ & 0.009 \\
\hline
\end{tabular}


students of grades 4 to 6 in Yiwu, a county-level city of Zhejiang Province, China [16]. The prevalence of myopia was found to be positively associated with grade and age. For the intervention of myopia, spectacles and contact lenses are considered as the mainstay to improve distance vision [9]. Pharmacological intervention includes nonselective muscarinic antagonist atropine and the M1 receptor-specific antagonist pirenzepine, which are also used to control myopia [17, 18], whereas refractive surgeries including keratorefractive procedures and intraocular procedures are used to correct refractive error [19-21]. Although the symptoms of myopia can be alleviated with those management practices, the risk of complications from potentially blinding conditions such as retinal detachments increase with the longer AL associated with high myopia [7, 22]. The prevention or delay of myopia by controlling environmental and genetic risk factors at the early age should be the priority for myopia control.

Parents having no myopia were identified to be a protective factor for myopia, suggesting hereditary factors may play an important role in myopia. Verhoeven et al. had identified multiple susceptibility loci for refractive error and myopia [11]. Multiple studies have suggested the family history of myopia was significantly associated with myopia $[23,24]$. In our study, adjusting the height of desks and chairs according to the changing height was also shown to be a protective factor, possibly due to the rapid change of stature in students of this age. The prevalence and the associations should be interpreted with caution because of the several limitations in this study. First, because of the relatively small sample size, some variates may not show a significant difference between myopic students and normal students, such as outdoor activities. Second, recall bias could exist in this cross-sectional study; hence, a longitudinal cohort trial was needed to further confirm the associations. Third, only two primary schools were included in this study, which led to a selection bias.

\section{Conclusions}

Our results showed that the prevalence of myopia among elementary school students was associated with environmental and hereditary factors.

\section{Abbreviations}

$\begin{array}{ll}\text { AL: } & \text { Axial length } \\ \text { CR: } & \text { Corneal radius } \\ \text { UCVA: } & \text { Uncorrected visual acuity } \\ \text { OR: } & \text { Odds ratio } \\ \text { CIs: } & \text { Confidence intervals. }\end{array}$

\section{Data Availability}

The data used to support the findings of this study are presented in the tables.

\section{Conflicts of Interest}

The authors declare that they have no conflicts of interest.

\section{Authors' Contributions}

Xin $\mathrm{Lu}$ and Congcong Guo contributed equally to this work.

\section{Acknowledgments}

The authors thank Liqin Ying from Taizhou Municipal Hospital for the eye examinations. This study was supported by the Science and Technology Project of Jiaojiang District, Taizhou City (No. 192031).

\section{References}

[1] I. G. Morgan, A. N. French, R. S. Ashby et al., "The epidemics of myopia: aetiology and prevention," Progress in Retinal and Eye Research, vol. 62, pp. 134-149, 2018.

[2] C. Jan, L. Li, L. Keay, R. S. Stafford, N. Congdon, and I. Morgan, "Prevention of myopia, China," Bulletin of the World Health Organization, vol. 98, no. 6, pp. 435-437, 2020.

[3] B. A. Holden, T. R. Fricke, D. A. Wilson et al., "Global prevalence of myopia and high myopia and temporal trends from 2000 through 2050," Ophthalmology, vol. 123, no. 5, pp. 1036-1042, 2016.

[4] I. G. Morgan, K. Ohno-Matsui, and S.-M. Saw, "Myopia," The Lancet, vol. 379, no. 9827, pp. 1739-1748, 2012.

[5] I. Morgan and K. Rose, "How genetic is school myopia?" Progress in Retinal and Eye Research, vol. 24, no. 1, pp. 1-38, 2005.

[6] M. He, F. Xiang, Y. Zeng et al., "Effect of time spent outdoors at school on the development of myopia among children in China," JAMA, vol. 314, no. 11, pp. 1142-1148, 2015.

[7] V. J. M. Verhoeven, K. T. Wong, G. H. S. Buitendijk, A. Hofman, J. R. Vingerling, and C. C. W. Klaver, "Visual consequences of refractive errors in the general population," Ophthalmology, vol. 122, no. 1, pp. 101-109, 2015.

[8] J. W. L. Tideman, M. C. C. Snabel, M. S. Tedja et al., “Association of axial length with risk of uncorrectable visual impairment for Europeans with myopia," JAMA Ophthalmology, vol. 134, no. 12, pp. 1355-1363, 2016.

[9] P. N. Baird, S. M. Saw, C Lanca et al., "Myopia," Nature Reviews Disease Primers, vol. 6, no. 1, p. 99, 2020.

[10] M. S. Tedja, R. Wojciechowski, P. G. Hysi, N. Eriksson, N. A. Furlotte, and V. J. M. Verhoeven, "Genome-wide association meta-analysis highlights light-induced signaling as a driver for refractive error," Nature Genetics, vol. 50, no. 6, pp. 834-848, 2018.

[11] V. J. Verhoeven, P. G. Hysi, R Wojciechowski et al., "Genomewide meta-analyses of multiancestry cohorts identify multiple new susceptibility loci for refractive error and myopia," Nature Genetics, vol. 45, no. 3, pp. 314-318, 2013.

[12] P. G. Hysi, H. Choquet, H. Choquet et al., "Meta-analysis of 542,934 subjects of European ancestry identifies new genes and mechanisms predisposing to refractive error and myopia," Nature Genetics, vol. 52, no. 4, pp. 401-407, 2020.

[13] J. W. L. Tideman, Q. Fan, J. R. Polling et al., "When do myopia genes have their effect? Comparison of genetic risks between children and adults," Genetic Epidemiology, vol. 40, no. 8, pp. 756-766, 2016.

[14] E. Mountjoy, N. M. Davies, D. Plotnikov et al., "Education and myopia: assessing the direction of causality by mendelian randomisation," BMJ, vol. 361, p. k2022, 2018.

[15] Y. Hu, X. Ding, X. Guo, Y. Chen, J. Zhang, and M. He, "Association of age at myopia onset with risk of high myopia 
in adulthood in a 12-year follow-up of a Chinese cohort," JAMA Ophthalmology, vol. 138, no. 11, pp. 1-6, 2020.

[16] J. Wang, G. S. Ying, X. Fu, R. Zhang, J. Meng, and F. Gu, "Prevalence of myopia and vision impairment in school students in Eastern China," BMC Ophthalmology, vol. 20, no. 1, p. 2, 2020.

[17] P. Ganesan and C. F. Wildsoet, "Pharmaceutical intervention for myopia control," Expert Review of Ophthalmology, vol. 1, no. 5, pp. 759-787, 2010.

[18] S. L. Pineles, R. T. Kraker, D. K. VanderVeen et al., "Atropine for the prevention of myopia progression in children," Ophthalmology, vol. 124, no. 12, pp. 1857-1866, 2017.

[19] R. Anderle and J. Ventruba, "The current state of refractive surgery," Collegium Antropologicum, vol. 37, no. 1, pp. 237241, 2013.

[20] A. Meduri, S. Z. Scalinci, M. Morara et al., "Effect of basic fibroblast growth factor in transgenic mice: corneal epithelial healing process after excimer laser photoablation," Ophthalmologica, vol. 223, no. 2, pp. 139-144, 2009.

[21] A. Meduri, L. Scorolli, S. Scalinci et al., "Effect of the combination of basic fibroblast growth factor and cysteine on corneal epithelial healing after photorefractive keratectomy in patients affected by myopia," Indian Journal of Ophthalmology, vol. 62, no. 4, pp. 424-428, 2014.

[22] D. I. Flitcroft, "The complex interactions of retinal, optical and environmental factors in myopia aetiology," Progress in Retinal and Eye Research, vol. 31, no. 6, pp. 622-660, 2012.

[23] W. Low, M. Dirani, G. Gazzard et al., "Family history, near work, outdoor activity, and myopia in Singapore Chinese preschool children," British Journal of Ophthalmology, vol. 94, no. 8, pp. 1012-1016, 2010.

[24] L. A. Jones, L. T. Sinnott, D. O. Mutti, G. L. Mitchell, M. L. Moeschberger, and K. Zadnik, "Parental history of myopia, sports and outdoor activities, and future myopia," Investigative Opthalmology \& Visual Science, vol. 48, no. 8, pp. 3524-3532, 2007. 